\title{
Assessment of Plasma Homocysteine Level among Sudanese Rheumatoid Arthritis Patients
}

\author{
Slsbeel Mohammed Elhassan Alrade ${ }^{1^{*}}$ and Amira Ahmed Khalid Humeida ${ }^{2}$ \\ ${ }^{1}$ Department of Hematology, Faculty of Medical Laboratory Sciences, AL-Neelain University, Khartoum, Sudan \\ ${ }^{2}$ Assistant Professor, Department of Pathology, Faculty of Medicine, AL-Neelain University, Khartoum, Sudan \\ Received 06 Nov 2017, Accepted 07 Jan 2018, Available online 16 Jan 2018, Vol.6 (Jan/Feb 2018 issue)
}

\begin{abstract}
Homocysteine (Hcy) is a four-carbon amino acid with a free thiol group, which is formed by demethylation of methionine, together with the help of some vitamins (B12, B6 and folate). The mechanisms responsible for hyperhomocysteinaemia in rheumatoid arthritis (RA) are not clear. However, drugs such as methotrexate has shown to have an effect on homocysteine metabolism, and interfers with vitamin metabolism and absorption. The aim of study was to assess plasma homocysteine level among Sudanese rheumatoid arthritis patients and to correlate homocysteine level with various types of treatment. A case control study was performed in Khartoum state, Sudan. A total of 60 Sudanese were enrolled in this study (30 RA patients and 30 healthy controls), 6 (20\%) were males and 24 (80\%) were females. Homocysteine levels were assessed using A15 analyzer. The consents were obtained from patients and it was approved by ethical committee of AL Neelain University. The mean $\pm S D$ was determined and then compared between cases group and controls group by calculating the P.value, the data was analyzed using SPSS. Homocysteine was found to be increased in 26 (86.7\%) out of 30 RA cases. There was a significant difference in homocysteine level ( $P$-value: $0.000)$ between cases and controls; with a mean of 24.6, $15.06 \mu \mathrm{mol} / \mathrm{L}$; respectively. There was a significant in homocysteine levels with different types of treatment (P-value: 0.000). Plasma homocysteine level increases in Rheumatoid arthritis.
\end{abstract}

Keywords: Plasma homocysteine, RA.

\section{Introduction}

\section{Homocysteine}

Homocysteine (Hcy) is a four-carbon amino acid with a free thiol group, which is formed by demethylation of methionine, an essential amino acid derived from diet. $^{(1)}$

Normal total homocysteine (tHcy) concentration range from 5-15 $\mu$ mol/L during fasting state. Hyperhomocystenemia (HHcy) has been classified into mild (plasma tHcy concentration of $15-30 \mu \mathrm{mol} / \mathrm{L}$ ), intermediate (plasma tHcy concentration of 31$100 \mu \mathrm{mol} / \mathrm{L}$ ), and sever (plasma tHcy concentration $100 \mu \mathrm{mol} / \mathrm{L})^{(2)}$. Both acquired and genetic factors can have an impact on plasma tHcy. Male gender, aging, smoking, impaired renal function, and some medication such as corticosteroid and cyclosporine are some examples of acquired causes and classic homocytinuria and C677T homozygote mutation of 5,10methylenetetrahydrofolate reductase (MTHFR) are the main genetic ones. Vitamin B12, vitamin B6, and folate,

*Corresponding author's ORCID ID: 0000-0002-6400-4612 DOI: https://doi.org/10.14741/ijmcr.v6i01.10910 which are all derived from dietary origins, are the three main cofactors in Hcy metabolism. Deficiencies in these supplements are more prevalent in the most developing countries and may account for many cases of moderate hyperhomocysteinemia $^{(1)}$

\section{Rheumatoid arthritis (RA)}

Rheumatoid arthritis (RA) is an autoimmune disease where the body's immune system mistakenly attacks the synovium, which is a thin membrane that lines the joints. It causes chronic inflammation that leads to pain, swelling and stiffness. Over time, RA can damage bones and joints leading to disability.

RA usually affects joints on both sides of the body equally, which means that if a joint on one side is affected, the same joint on the opposite side is affected. Wrists, Fingers, knees, feet, and ankles, and elbows are most oftenly affected. RA can also affect other organs, therefore, people who have RA are at higher risk for other diseases which include; heart disease, diabetes, and osteoporosis. $^{(3)}$

In patients with RA, life expectancy is 3-10 years shorter than normal population. ${ }^{(4)}$ Cardiovascular $77 \mid$ Int. J. of Multidisciplinary and Current research, Vol.6 (Jan/Feb 2018) 
diseases including vascular disease of the brain are responsible of $40-50 \%$ of the death in RA. ${ }^{(5)}$ Intima-media thickness (IMT) and plaque frequency were increased in subclinical atherosclerosis studies, and this may be a sign for early-onset atherosclerosis in RA. ${ }^{(6)}$

High levels of homocysteine are commonly found in patients with rheumatoid arthritis (RA), thus accounting, at least partially, for the high rate of mortality from cardiovascular events in these subjects. (7) The mechanisms responsible for hyperhomocysteinaemia in RA are not clear. However, drugs such as methotrexate and sulfasalazine affect homocysteine metabolism, and interfere with vitamin metabolism and absorption $^{(8)}$.Furthermore, an increased use or accelerated catabolism of vitamin $\mathrm{B} 6$ has been shown in chronic inflammatory diseases, particularly $\mathrm{RA}^{(9)}$.

\section{Materials and Methods}

This study was a case control study, which was conducted in Khartoum, Sudan, Military hospital, during the time period from May to October 2017, and consents were obtained from patients and it was approved by ethical committee of AL Neelain University.

Sixty samples were included in this study for assessment of homocysteine level in patients with rheumatoid arthritis (6 males and 24 females). Their ages range was 20-60 years, age and sex were matched controls group were 30 samples from normal individuals. Patients group included in the study were only old cases with RA who were on treatment. Patients with diabetes, renal failure, cardiac disease, liver failure, malignancies, transplantation, smokers, alcoholic and new cases receiving no treatment were excluded from this study.

$3 \mathrm{ml}$ of blood were collected from each subject by clean venous puncture collected in EDTA anticoagulant tubes. Blood samples were centrifuged for $5 \mathrm{~min}$ at $3 \mathrm{rpm}$ and were kept at $-20^{\circ} \mathrm{C}$ until use, samples were analyzes by using Biosystem reagents (homocysteine enzymatic cycling) and fully automated Biosystem device (A15) and homocysteine levels was assessed. The mean \pm SD was determined and then compared between the case group and control group by calculating the P.value, the data were analyzed using SPSS.

\section{Result}

This study showed that the levels of homocysteine were increased in 26 (86.7\%) out of 30 RA cases. There was a difference in homocysteine level ( $P$-value: 0.000) between cases and controls; with a mean of 24.6, 15.05 $\mu \mathrm{mol} / \mathrm{L}$; respectively (Figure 1 ). There was an association between RA and Homocysteinemia (P-value 0.000) (figure 2). However there was a difference in homocysteine level between the types of treatment, VitB (mean $17.81 \pm 8.72$ $\mu \mathrm{mol} / \mathrm{L}: \quad \mathrm{P} 0.001), \quad \mathrm{MTX}$ (mean $31.60 \pm 7.72 \mu \mathrm{mol} / \mathrm{L}$ : P0.000), MTX+folic acid (mean 24.03 $\pm 5.89 \mu \mathrm{mol} / \mathrm{L}$ : P0.000), HCQ $200 \mathrm{mg}$ (mean 29.63 $\pm 3.84 \mu \mathrm{mol} / \mathrm{L}:$ P0.000) Table 1.

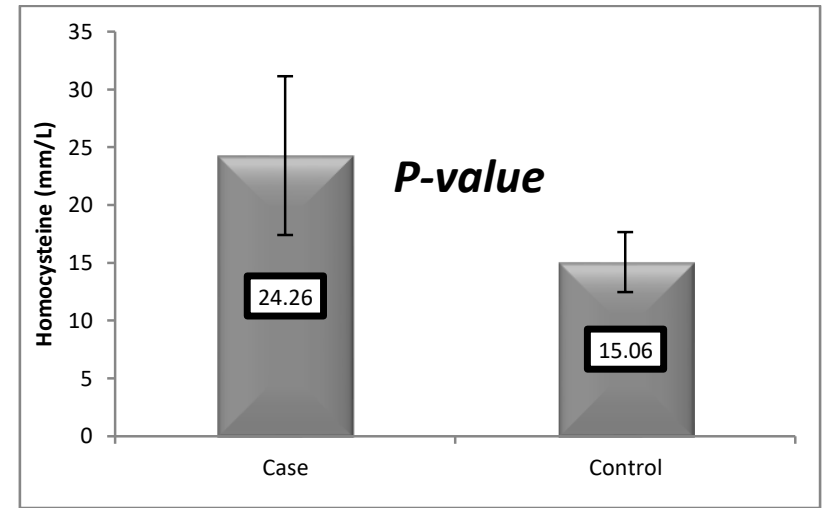

Figure 1: Comparison of the mean of Homocysteine levels ( $\mu \mathrm{mol} / \mathrm{L}$ ) among cases and controls

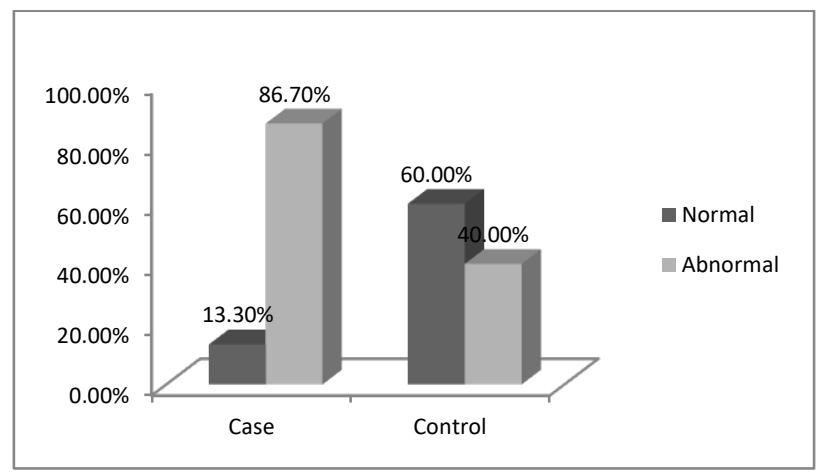

Figure 2: Association of RA and Homocysteinemia

Table 1: Homocysteine levels $(\mu \mathrm{mol} / \mathrm{L})$ with different types of treatment

\begin{tabular}{|c|c|c|}
\hline Types of treatment & Mean \pm SD & P-value \\
\hline Vit B & $17.81 \pm 8.72$ & 0.001 \\
\hline MTX & $31.60 \pm 7.72$ & 0.000 \\
\hline MTX+folic acid & $24.03 \pm 5.89$ & 0.000 \\
\hline HCQ 200mg & $29.63 \pm 3.84$ & 0.000 \\
\hline
\end{tabular}

\section{Discussion}

Hyperhomocysteinaemia is known to be a risk factor for vascular occlusive diseases, elevated levels of plasma homocysteine have been found in patients suffering from peripheral vascular occlusions, such as coronary artery disease, cerebral vascular accidents and deep-vein thrombosis as well as those suffering from ocular vascular occlusions such as retinal vein and retinal artery and anterior ischemic optic neuropathy ${ }^{(10)}$.

The present study revealed an increase in plasma homocysteine levels in RA cases as compared to non- RA controls, analysis of these data showed that plasma homocysteine levels showed significant difference between cases and controls, the majority of cases showed abnormal high homocysteine level (86.7\%).

The study finding of high concentrations of plasma homocysteine levels was found to agree with another study done in 2015 by Dragan, et al who studied 60 patients with RA and reported high concentration of 78 | Int. J. of Multidisciplinary and Current research, Vol.6 (Jan/Feb 2018) 
plasma homocysteine level (mean $11.79 \pm 3.72 \mu \mathrm{mol} / \mathrm{L}$ : $\mathrm{P}<0.01)^{(11)}$

The study finding of high concentrations of Hcy levels significantly in methotrexate treated patients were found to agree with another study done in 2015 by Emrullah HAYTA, et al who studied 58 female with RA and reported that the levels of Hcy was found to be significantly high in methotrexate treated patients (mean $16.88 \pm 6.84 \mu \mathrm{mol} / \mathrm{L}$ : P0.001). ${ }^{(12)}$

\section{Conclusion}

This study, based on a small number of subjects, found significantly higher levels of homocysteine in RA patients. Whose plasma homocysteine level was influenced by the type of treatment of RA. A further larger sample size a study is needed in order to define the role of Homocyteine level among RA patients by using one type of treatment.

\section{References}

[1]. Christopbor R, Nagaraja D, Shanker SK, Homocysteine and Cerebral stork in developing countries. Curr Med Chem. 2007:14(22):2393-401.

[2]. www.eMedicinHealth.com (Medical Author: Benjamin Wedro, MD, FACEP, FAAEM).

[3]. Maini RN, Zvaifler NJ. Rheumatoid arthritis and spondyloarthropathy. In: Klippel JH, Dieppe PA, editors. Rheumatology. St Louis: Mosby: 1994.P.3:1,1-40:1.

[4]. Kerekes G, Szekanecz Z, Der H, Sandor Z, Lakos G, Muszbek L. Endothelial dysfunction and atherosclerosis in rheumatoid arthritis: a multiparametric analysis using imaging techniques and laboratory markers of inflammation and autoimmunity. J Rheumatol 2008:35:398-406.
[5]. Kapetanovic MC, Lindqvist E, Simonsson $M$, Geborek $P$, Saxne T, Eberhardt K. Prevalence and predictive factors of comorbidity in rheumatoid arthritis patients monitored prospectively from disease onset up to 20 years: lack ofassociation between inflammation and cardiovascular disease. Scand J Rheumatol 2010:39:353-9.

[6]. Ciftci O, Yilmaz S, Topcu S, Caliskan M, Gullu H, Erdogan D. Impaired coronary microvascular function and increased intima-media thickness in rheumatoid arthritis. Atherosclerosis 2008; 198:332-7.

[7]. KroghJensen $M$, Ekelund $S$, Svendsen L. Folate and homocysteine status and haemolysis in patients treated with sulphasalazine for arthritis. Scand J Clin Lab Invest1996; 56:421-9

[8]. Haagsma CJ, Blom HJ, van Riel PL, van't Hof MA, Giesendorf BA, van Oppenraaij-Emmerzaal D. Influence of sulphasalazine, methotrexate, and the combination of both on plasma homocysteine concentrations in patients with rheumatoid arthritis. Ann Rheum Dis1999; 58:79-84.

[9]. McCarty MF. Increased homocysteine associated with smoking, chronic inflammation, and aging may reflect acute-phase induction of pyridoxal phosphatase activity. Med Hypoth2000; 55:289-93.

[10]. Goldstein,M. I.Leibovitc, I. Yeffimov, S. Gavendo, B.A. Sela and A. Loewenstein. (2004). Hyperhomocysteinemia in patients with diabetes mellitus with and without diabetic retinopathy. Eye. 18: 460-465.

[11].Dragan Vasiljevic, Aleksandra Tomic-Lucic, Sandra Zivanovic, Mirjana Milosavljevic, Snezana Radovanovic, Nebojsa Andjelkovic, Dragan Djuric, Mirjiana Veselinovic, published online:2015-10-14/ DOL: https://doiorg/10.1515/sjecr-2015-0027.

[12]. Emrullah HAYTA, Sami HiZMETLi, Mehmet Haydar ATALAR, Ziynet CINAR. J Rheumatology 10.5606/Arch Rheumatol.2015.4249:214-220. 